\title{
An efficient computer forensics selective imaging model
}

\begin{abstract}
Selective imaging is a new concept in computer forensics. It is used for collecting only the data that is relevant to the crime and helps in improves the scalability of the investigation process. However, the current selective imaging approaches directly image the identified data without considering their offsets on the targeted user storage. This paper investigates the impact of the relevant data offsets on the efficiency of the selective imaging process. A practical selective imaging model is presented which includes a digital evidence ordering algorithm (DEOA) for ordering the selected relevant data items. The proposed selective imaging model has been implemented and evaluated in different types of storage devices. The evaluation result shows that even if our proposed algorithm has a small efficiency negative impact before the imaging process starts; it has a large positive effect on the efficiency of the selective imaging process itself.
\end{abstract}

Keyword: Computer forensics; Digital evidence; Selective imaging; Efficiency; Ordering algorithm 